\title{
Redundancy and viewing time
}

\author{
ALLEN GOLDBLATT \\ Tel Aviv University, Tel Aviv, Israel \\ and \\ J. N. EACKER \\ Whitman College, Walla Walla, Washington 99362
}

\begin{abstract}
An experiment was performed to test the effects of redundancy in visual patterns on looking time. A series of 308 by 8 black and white checkerboards were constructed which differed in the number of choice points used in their construction. Five levels of redundancy were used. Twenty subjects were told to look at each slide until they lost interest in it. A record was kept of the amount of time spent viewing each slide. The data were analyzed and it was found that looking time increased as redundancy decreased, but, as redundancy decreased beyond a certain point, looking time failed to increase.
\end{abstract}

Many experiments have dealt with the effects of visual patterns on exploratory behavior. Some of these experiments have been concerned with the amount of time a subject views different visual patterns. The types of visual patterns used have varied from photographs (Leckart \& Bakan, 1965) to randomized patterns (Berlyne, 1957, 1963; Day, 1966). In general, these patterns have been ordered along such parameters as complexity, redundancy, and symmetry.

Berlyne (1957) used a series of patterns which varied in complexity and he found a positive relationship between complexity and looking time. He stated that complexity is related to relative entropy, which is the inverse of redundancy. He tested the effects of varying redundancy and concluded that looking time increases with relative entropy if maximum entropy is held constant. Relative entropy was defined as the ratio of the amount of information received from a figure to the maximum that could be received from a figure with the same quantity of material.

A major criticism of this work is that both the metron content and the logon content of the stimuli were confounded within each series. Metron content is the variation in units along each attribute, while logon content is the different types of variation or attributes (Brown \& Michels, 1966). It is not known whether the increase in looking time reported by Berlyne is due to the reduction of redundancy or to the increase in complexity of the stimuli. A method which can be used to determine if looking time increases with decreasing redundancy and independently of complexity is to construct a series of slides which vary only in their relative redundancy. Such slides would hold the metron content constant while varying the logon content. With slides it is possible to test more clearly whether

This experiment was done as a partial requirement for the BA degree from Whitman College. R. E. Lubow sponsors this paper and takes full editorial responsibility for its content. there is an inverse variation between redundancy and looking time.

For this experiment a series of slides was constructed in which only relative redundancy was varied; it was hypothesized that looking time would increase as redundancy decreased.

\section{METHOD}

\section{Subjects}

The subjects were 20 students selected at random from the student population of Whitman College. There were 9 males and 11 females.

\section{Stimulus Patterns}

The stimuli were slides of 8 by 8 black and white checkerboard patterns photographed against a flat black background. There were 30 slides, divided into 6 slides for each of five levels of redundancy. The levels of redundancy were distinguished by the number of choice points used in their construction. The number of choice points was $4,8,16,32$, and 64 (complete randomness). Complete redundancy would have been a simple alternating 8 by 8 checkerboard.

The method of stimulus construction was as follows: For the slides constructed with four choice points, the basic unit was a 4 by 4 simple alternating checkerboard. The color of the upper left-hand cell for each of the four units was determined at random, which then fixed the colors for the rest of the cells in that unit. Thus, four simple alternating checkerboards were used in the most redundant pattern.

For the slides with eight choice points, a 4 by 2 alternating checkerboard was the basic unit. Slides with 16 choice points had a 2 by 2 simple alternating checkerboard as the basic unit. Slides with 32 choice points used a pair of cells as the basic unit. In each case, the color of the upper left-hand square was determined at random, thus determining the pattern of the basic unit. For the slides with 64 choice points each square was determined at random.

\section{Apparatus}

The apparatus consisted of a Kodak Carousel slide projector, a screen $8 \mathrm{ft}$ from the projector, and interval timer (GrasonStadler), a Hunter printout counter, and a control box with an "expose" button and a "change" button. The testing room was $8 \times 10 \mathrm{ft}$ and contained only the projector and screen, the control box, and a chair. The apparatus was arranged so that 
when a subject pressed the expose button, a slide appeared and the timer was activated. When the change button was pressed, the slide was replaced by a clear slide, the timer was stopped, and the amount of time the stimulus slide was projected was printed.

\section{Procedure}

The 30 stimulus patterns were randomly assigned to six groups of five slides each, with the restriction that each group should contain one slide for each degree of redundancy. Subjects were tested individually. They were seated next to the control box and were told to read the instructions next to them. Subjects were instructed to press the button labeled "expose" and to look at a slide until they lost interest in it. When they were ready to look at a new slide, they were to repeat the procedure. "Practice" was written on two practice slides which were used to adapt the subject to the apparatus and procedure. Subjects were not told that they were being timed and it was emphasized that they would not be tested afterwards. The six groups of slides were randomized after each subject.

\section{RESULTS}

The mean looking time for each degree of redundancy is presented in Figure 1, where it will be observed that looking time increases as redundancy decreases up to the 32 choice point level; a decrease in

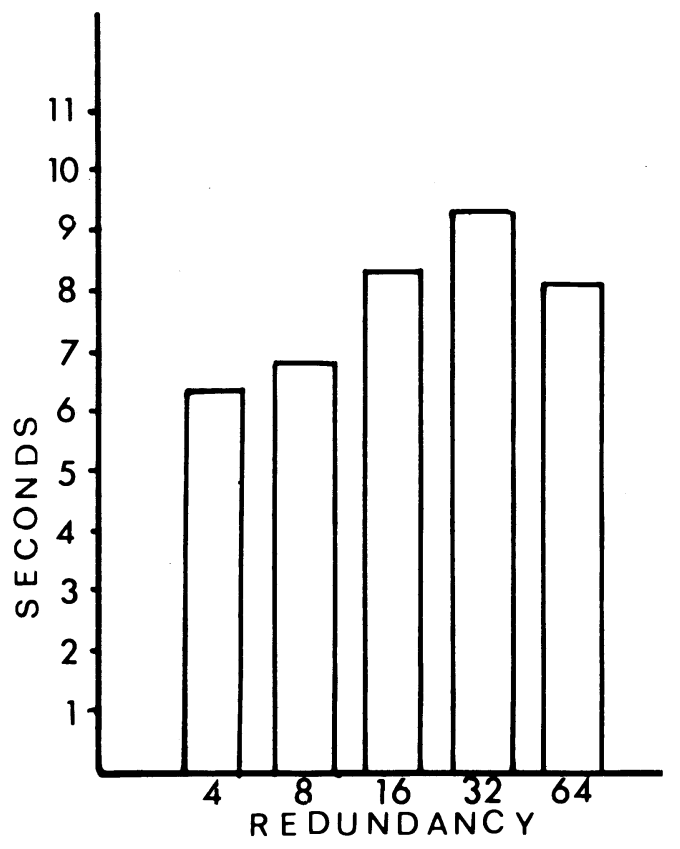

Figure 1. Mean looking time in seconds as a function of the degree of redundancy. looking time occurred between 32 and 64. An analysis of variance on these data showed that the observed effect of redundancy reached statistical significance $(\mathrm{F}=2.69, \mathrm{df}=4 / 76, \mathrm{p}<.05)$. Individual comparisons (Newman-Keuls) showed that Levels 4 and 8 differed from Level $32(p<.05)$. All other comparisons failed to reach significance.

\section{DISCUSSION}

The results indicate that looking time increases as redundancy decreases, but that beyond a certain level it does not. These results are in agreement with Berlyne (1957), who found that looking time increases as relative entropy increases. A major difference between this study and that of Berlyne is that, whereas he manipulated both relative and absolute entropy, in the present study only relative entropy was manipulated. The results are also in agreement with those obtained in experiments where only complexity was manipulated. For example, both Day (1966) and Leckart and Bakan (1965) found that looking time increased with increasing complexity but that at high levels of complexity there was a decrease in looking time. It appears, therefore, that a decrease in redundancy produces the same changes in looking behavior as does an increase in complexity.

There is a need for the standardization of stimuli along single dimensions, that is, stimuli which vary in either logon or metron content. With respect to metron content, polygons generated by Attneave's Method 1 would be adequate (Attneave \& Arnoult, 1956). With respect to logon content, the checkerboard method described above can generate patterns within any given matrix which can extend from complete redundancy to complete randomness. By choosing either polygons or checkerboard patterns, an investigator can examine either redundancy or complexity independently of each other.

\section{REFERENCES}

Attneave, F., \& Arnoult, M. The quantitative study of shape and pattern perception. Psychological Bulletin, 1956, 53, 452-471.

BERLYNE, D. E. Conflict and information-theory variables as determinants of human perceptual curiosity. Journal of Experimental Psychology, 1957, 53, 399-404.

BERLYNE, D. E. Complexity and incongruity variables as determinants of exploratory choice and evaluative ratings. Canadian Journal of Psychology, 1963, 17, 247-290.

Brown, D. R., \& Michels, K. M. Quantification procedures, stimulus domains and discrimination difficulty. Perceptual and Motor Skills, 1966, 22, 423-428.

DAY, H. Looking time as a function of stimulus variables and individual differences. Perceptual and Motor Skills, 1966, 22, 423-428.

LeCKART, B. T., \& BAKAN, P. Complexity judgments of photographs and looking time. Perceptual and Motor Skills, 1965, 21, 16-18.

(Received for publication October 6, 1976.) 